\title{
CONSUMO HÍDRICO E ENERGÉTICO NA IRRIGAÇÃO DO CAFÉ NO TRIÂNGULO MINEIRO E OESTE DA BAHIA
}

\author{
Rodrigo Otávio Câmara Monteiro ${ }^{1}$, Rubens Duarte Coelho ${ }^{2}$ Marconi Batista Teixeira $^{3}$ \\ ${ }^{1}$ Instituto Federal de Educação, Ciência e Tecnologia do Rio Grande do Sul- IFRS. Av. Osvaldo Aranha 540 - $95700-000$ - \\ Bento Gonçalves - RS. *Autor para correspondência: rodrigo.monteiro@ bento.ifrs.edu.br \\ ${ }^{2}$ Universidade de São Paulo - USP/ESALQ, Caixa Postal 9 - 13418-900 - Piracicaba - SP, Brasil, rdcoelho@esalq.usp.br \\ ${ }^{3}$ Instituto Federal de Educação, Ciência e Tecnologia de Goiás, Rod. Sul Goiana Km 01 - 75901-970, Zona Rural- Rio Verde - \\ GO, Brasil
}

\section{RESUMO}

Com o aumento da pressão pela racionalização da exploração agrícola a caminho da sustentabilidade e a incorporação de novas tecnologias, tornam-se necessários o domínio, o conhecimento e o manejo dos principais fatores relacionados à produção, dentre os quais o consumo hídrico e energético. Este estudo, portanto, foi realizado com o objetivo de avaliar as necessidades de água e o consumo de energia (elétrica e diesel) para os quatro primeiros anos do desenvolvimento do café, no plantio tradicional, irrigado por pivô central, pivô central com emissores tipo LEPA e por gotejamento. As avaliações foram baseadas em informações edafoclimáticas das regiões do Triângulo Mineiro e do Oeste da Bahia. A lâmina de água aplicada pelo sistema de gotejamento, em todo o período, foi mais de $50 \%$ inferior à lâmina aplicada pelos outros sistemas, além de apresentar uma economia média de energia elétrica em torno de $38 \%$. O bombeamento de água usando energia elétrica para irrigação do cafeeiro, nas regiões estudadas, é significativamente mais econômico do que o acionamento usando motor diesel.

Palavras-chave: cafeicultura, sistemas de irrigação, energia elétrica e diesel

\section{WATER AND ENERGY CONSUMPTION ON IRRIGATION FOR COFFEE CROP AT 'TRIÂNGULO MINEIRO' AND WESTERN BAHIA REGIONS, BRAZIL}

\begin{abstract}
Due to the increasing pressure to rationalize farm production for sustentability and the adoption of new technologies one has to know the main production management factors, such as water and energy consumption. This paper evaluates the crop water demand and energy (electrical and diesel)consumption during the first four years of coffee development in traditional tillage, by central pivot, central pivot using LEPA sprinkler and drip irrigation. The evaluations were based in soil and climatic data of the "Triangulo Mineiro", Minas Gerais State, and Western Bahia State areas, in Brazil.The water depth applied by drip irrigation system saved up to $50 \%$ of the one applied by the other irrigation systems, besides saving $38 \%$ of electric energy. Water pumping by electric energy was significantly more economical than water pumping by diesel fuel.
\end{abstract}

Key words: coffee crop, irrigation systems, electric and diesel energy 


\section{INTRODUÇÃO}

Nos últimos 25 anos o cafeeiro vem sendo cultivado em regiões com extensos períodos de deficiência hídrica, sendo essencial a irrigação. Destacam-se nestes casos, as regiões do Triângulo Mineiro e Alto Paranaíba em Minas Gerais, Norte do Espírito Santo e as regiões Sul e Oeste da Bahia. Resultados obtidos por pesquisadores (Gomes et al., 2007; Rezende et al., 2006; Silva et al., 2003; Lima et al., 2008) têm justificado a adoção da técnica de irrigação em função dos incrementos proporcionados à produtividade do cafeeiro.

A determinação da demanda de água pelo cafeeiro (ETc), assim como os coeficientes utilizados no manejo da irrigação tem sido um grande desafio para os pesquisadores em todo o Brasil e no mundo, os quais têm procurado caracterizar regionalmente esses fatores. Com a criação do Programa Nacional de Pesquisa e Desenvolvimento da Cafeicultura (PNP\&D/Café), pesquisas com irrigação foram intensificadas, visando apoiar $\mathrm{o}$ suporte técnico para que haja uma tecnologia fundamentada e propulsora de lucratividade e sustentabilidade aos cafeicultores (Silva $e t$ al., 2002).

A irrigação, todavia, é considerada uma tecnologia altamente consumidora de energia, tendo destaque especial no custo total de produção (Monteiro et al., 2007). Sabe-se que não existe um sistema ideal de irrigação em relação à utilização de energia. Vários fatores determinam a escolha de um sistema de irrigação, dentre os quais, o clima, o solo, a disponibilidade e a qualidade de água, as culturas e o consumo de energia e de água. Todos estes fatores devem ser devidamente considerados em uma decisão econômica e ambiental, visando definir o sistema de irrigação mais adaptado às características de cada local.
Neste contexto, este estudo foi realizado com o objetivo de avaliar as necessidades de água e o consumo de energia (elétrica e diesel) para os quatro primeiros anos de desenvolvimento do café (2006-2009) no sistema de plantio tradicional e irrigado por três sistemas de irrigação: pivô central $\left(\mathrm{SI}_{1}\right)$, pivô central com emissores tipo LEPA (SI2) e por gotejamento (SI3), para as regiões do Triângulo Mineiro e do Oeste da Bahia.

\section{MATERIAL E MÉTODOS}

O estudo foi realizado em duas propriedades com café irrigado, localizadas, respectivamente, em Patrocínio - MG (latitude de $18^{\circ} 56^{\prime} \mathrm{S}$, longitude de $46^{\circ} 59^{\prime} \mathrm{W}$ e altitude de 965,52 m) e Barreiras - BA (latitude de $12^{\circ} 9^{\prime} \mathrm{S}$, longitude de $44^{\circ} 59^{\prime} \mathrm{W}$ e altitude de 452,23 m). Os solos das regiões estudadas são Latossolos Vermelhoamarelos.

O perfil de ambos os solos foi dividido em camadas sendo a primeira de 0 a $0,20 \mathrm{~m}$ e a segunda de 0,20 a $0,60 \mathrm{~m}$ de profundidade. A caracterização física é apresentada na Tabela 1.

As umidades na capacidade de campo e no ponto de murcha permanente foram determinadas a partir da curva de retenção de água no solo. A densidade global do solo foi obtida usando anéis volumétricos com volume conhecido com amostras sem deformação. As amostras foram secas pelo método da estufa. Após esse processo, pesou-se novamente, obtendo-se a massa de sólidos. A densidade média do solo foi obtida pela relação entre massa de sólidos e volume, com três repetições. Para a análise granulométrica, as frações do solo foram separadas previamente. Utilizou-se o peneiramento, para as frações areia grossa e areia fina, e a sedimentação, para as frações silte e argila. (EMBRAPA, 1997). 
Tabela 1. Caracterização físico-hídrica dos Latossolos Vermelho-amarelos das regiões do Triângulo Mineiro (Patrocínio-MG) e Oeste da Bahia (Barreiras-BA), nas camadas de 0 a $0,20 \mathrm{~m}$ e 0,20 a $0,60 \mathrm{~m}$.

\begin{tabular}{|c|c|c|c|c|c|c|}
\hline \multirow{2}{*}{$\begin{array}{l}\text { Camada } \\
\text { (m) }\end{array}$} & $\mathrm{CC}^{1}$ & $\mathrm{PMP}^{2}$ & \multirow{2}{*}{$\begin{array}{c}\mathrm{ds}_{\mathrm{s}}^{3} \\
\mathrm{~g} \mathrm{~m}^{-3}\end{array}$} & Areia & Silte & Argila \\
\hline & \multicolumn{2}{|c|}{$(\%)$} & & \multicolumn{3}{|c|}{$(\%)$} \\
\hline & \multicolumn{6}{|c|}{ Latossolo Vermelho Amarelo - Triângulo Mineiro } \\
\hline $0-0,20$ & 34 & 22,5 & 1,20 & 20 & 30 & 50 \\
\hline \multirow[t]{2}{*}{$0,20-0,60$} & 32,6 & 22,3 & 1,19 & 20 & 30 & 50 \\
\hline & \multicolumn{6}{|c|}{ Latossolo Vermelho Amarelo - Oeste da Bahia } \\
\hline $0-0,20$ & 11,8 & 4,5 & 1,67 & 79 & 3 & 18 \\
\hline $0,20-0,60$ & 11,8 & 4,5 & 1,78 & 79 & 3 & 18 \\
\hline
\end{tabular}

1-Umidade gravimétrica na capacidade de campo; 2-Umidade gravimétrica no ponto de murcha permanente; 3 densidade global do solo

A cultura utilizada foi o café $\mathrm{cv}$. Catuaí, sendo considerados os seguintes parâmetros: fator de disponibilidade hídrica de 0,4 , temperatura basal da cultura de 10,0 ${ }^{\circ} \mathrm{C}$, temperatura ótima para a cultura de 25,0 ${ }^{\circ} \mathrm{C}$ e salinidade máxima para a cultura de $10,0 \mathrm{dS} \mathrm{m} \mathrm{m}^{-1}$. Demais dados técnicos são apresentados na Tabela 2 . Os

coeficientes de cultura $(\mathrm{Kc})$, por estádio de desenvolvimento, foram ajustados de acordo com a variação climática de cada região, seguindo a metodologia proposta por Allen et al. (1998) (Tabela 2). Para as duas regiões estudadas utilizou-se os mesmos tratos culturais e espaçamentos $(3,80 \mathrm{~m}$ entre fileiras e $0,75 \mathrm{~m}$ entre plantas), diferenciando-se as características de solo e os fatores de correção da evapotranspiração.

Os coeficientes de localização (Ks) foram adotados conforme Keller \& Karmeli (1975). Para o sistema de irrigação por gotejamento $\left(\mathrm{SI}_{3}\right)$ os valores utilizados foram de 0,$99 ; 0,98 ; 0,96$ e 0,94 , nos anos 1 , 2, 3 e 4 do ciclo da cultura, respectivamente. Para os demais sistemas, foi adotado o valor unitário. O plantio, em ambas as áreas analisadas, foi considerado na mesma data, e com o mesmo conteúdo de água no solo.

Com base nos dados climáticos diários de cada região (Instituto Nacional de
Meteorologia - INMET), foi determinada, por meio do balanço hídrico, para os 4 anos de estudo, a lâmina a ser aplicada e o tempo de funcionamento do equipamento de irrigação utilizado. A estimativa da evapotranspiração de referência $\left(\mathrm{ET}_{0}\right)$ foi obtida pela equação de Penman-Monteith, recomendada pela FAO (Allen et al., 1998). A necessidade hídrica da cultura do café, para cada ano, em cada região, foi obtida, a partir da evapotranspiração da cultura (ETc) acumulada neste período. Com o programa IRRIGER - Gerenciamento e Engenharia de Irrigação (2006), foi realizado o estudo desde o plantio (2006) até a fase adulta da cultura (2009).

Foram adotadas para os sistemas de irrigação por pivô central $\left(\mathrm{SI}_{1}\right)$, pivô central com emissor LEPA $\left(\mathrm{SI}_{2}\right)$ e por gotejamento $\left(\mathrm{SI}_{3}\right)$, as relações de potência/área abrangida pelo conjunto motobomba de $\mathrm{SI}_{1}=2,0$; $\mathrm{SI}_{2}=1,5 \mathrm{e} \mathrm{SI}_{3}=1,2 \mathrm{cv}$ ha $^{-1}$, respectivamente. As áreas irrigadas de cada sistema de irrigação foram equivalentes a 100 ha. Foram consideradas, ainda, as uniformidades de Christiansen (CUC) de 85, 90 e 92\%, respectivamente, para os sistemas $\mathrm{SI}_{1}, \mathrm{SI}_{2}$, $\mathrm{SI}_{3}$, conforme Bonono (1999), Santinato e Fernandes (2002) e Souza (2000), além de uma eficiência de aplicação de água de $90 \%$. 
Tabela 2. Caracterização dos estádios da cultura do café (dias), coeficiente de cultura - Kc (adimensional), profundidade efetiva do sistema radicular (m) e área sombreada (\%) para as regiões do Triângulo Mineiro (TM) e Oeste da Bahia (OB).

\begin{tabular}{ccccc}
\hline $\begin{array}{c}\text { Estádio } \\
\text { fenológico }\end{array}$ & $\begin{array}{c}\text { Duração } \\
\text { (dias) }\end{array}$ & Kc & $\begin{array}{c}\text { Profundidade } \\
\text { efetiva de raízes } \\
(\mathrm{m})\end{array}$ & $\begin{array}{c}\text { Área sombreada } \\
(\mathrm{TM}-\mathrm{OB})(\%)\end{array}$ \\
\hline Plantio & 185 & 0,45 & 0,20 & 5,0 \\
Desenvolvimento & 180 & 0,55 & 0,30 & 10,0 \\
Ano 2 (TM) & 185 & 0,65 & 0,40 & 20,0 \\
Ano 2 (OB) & 180 & 0,70 & 0,45 & 25,0 \\
Ano 3 (TM) & 185 & 0,80 & 0,50 & 35,0 \\
Ano 3 (OB) & 180 & 0,85 & 0,60 & $40,0-45,0$ \\
Adulto & 365 & 0,90 & 0,60 & $50,0-55,0$ \\
\hline
\end{tabular}

Conhecido o somatório das lâminas aplicadas nos quatro primeiros anos de desenvolvimento da cultura e sua necessidade hídrica, foi possível determinar, para cada sistema de irrigação, os custos horários totais de energia elétrica, a partir do custo anual total do sistema elevatório (Eq. 01) e o número de horas de irrigação. O consumo energético para tais condições foi obtido respeitando-se as especificidades das tarifas (modalidades, horário fora de ponta, desconto noturno, períodos seco e úmido) (Brasil, 2000; Brasil, 2002) cobradas pelas concessionárias energéticas das regiões de estudo e, para a energia a diesel, o preço do diesel na propriedade rural. Abaixo, segue a metodologia de cálculo para as duas fontes energéticas:

$$
C A T=C A F+C A V
$$

Em que:

CAT - custo anual total do sistema elevatório (\$);

CAF - custo anual fixo do sistema elevatório

(\$);
CAV - custo anual variável do sistema elevatório (\$).

Para a determinação do custo anual fixo, foram utilizadas as equações 02 a 08 , conforme descrito na seqüência abaixo:

$$
C A F=\left(P_{i}-R_{i}\right) \cdot\left[\frac{j \cdot(1+j)^{n_{i}}}{(1+j)^{n_{i}}-1}\right]+R_{i} \cdot j
$$

Em que:

$\mathrm{P}_{\mathrm{i}}$ - valor inicial (\$) do i-ésimo componente do bem de capital;

$\mathrm{R}_{\mathrm{i}}$ - valor residual do i-ésimo componente; $\mathrm{j}$ - taxa de juros reais; $\mathrm{n}_{\mathrm{i}}$-vida útil do i-ésimo componente (anos).

$\mathrm{O}$ valor do investimento inicial do sistema elevatório foi obtido a partir da Eq. 03: 


$$
\text { InvIn }=V i_{C H}+V i_{C B}+\left\{V i_{R E} \text { ou } V i_{M C A}\right\}
$$

Em que:

$\mathrm{Vi}_{\mathrm{CH}}$ - valor inicial (\$) dos componentes hidráulicos instalados;

$\mathrm{Vi}_{\mathrm{CB}}$ - valor inicial (\$) da casa de bombas e estruturas anexas;

$\mathrm{Vi}_{\mathrm{RE}}$ - valor inicial (\$) da rede e equipamentos elétricos instalados (para

bombas hidráulicas acionadas por motores elétricos);

$\mathrm{Vi}_{\mathrm{MCA}}$ - valor inicial (\$) do(s) motor(es) à combustão e acessórios instalados (para bombas hidráulicas acionadas por motores à combustão).

Foram considerados os seguintes componentes hidráulicos $\left(\mathrm{Vi}_{\mathrm{CH}}\right)$ (Eq. 04):

Em que:

$\mathrm{Vi}_{\mathrm{ts}}$ - valor inicial (\$) da tubulação de sucção com os acessórios;

$\mathrm{Vi}_{\text {tr }}$ - valor inicial (\$) da tubulação de recalque com os acessórios;

$\mathrm{Vi}_{\mathrm{bh}}$ - valor inicial (\$) da bomba hidráulica com os acessórios;

$$
V i_{C B}=v u_{c c} \cdot A_{c b} \cdot f a
$$

Em que:

$\mathrm{Vi}_{\mathrm{CB}}$ - valor inicial (\$) da casa de bombas; $\mathrm{vu}_{\mathrm{cc}}$ - valor unitário $\left(\$ \mathrm{~m}^{-2}\right)$ da construção civil para obras de acabamento rústico ou normal; $\mathrm{A}_{\mathrm{cb}}$ - área da casa de bombas $\left(\mathrm{m}^{2}\right)$;
$\mathrm{Vi}_{\text {sich }}$ - valor (\$) dos serviços de instalação dos componentes hidráulicos.

$\mathrm{O}$ valor inicial da casa de bombas e estruturas anexas $\left(\mathrm{Vi}_{\mathrm{CB}}\right)$ foi obtido a partir da equação 05 :

$$
V i_{R E}=V i_{l a t}+V i_{e e}
$$

Em que:

$\mathrm{Vi}_{\text {lat }}$ - valor inicial (\$) da linha de alta tensão;

fa - fator de acréscimo para as obras das estruturas anexas (ex: pavimentação da área externa próxima à casa de bombas).

Para o valor inicial da rede e equipamentos elétricos $\left(\mathrm{Vi}_{\mathrm{RE}}\right), \quad$ foi considerado (Eq. 06):

$\mathrm{Vi}_{\mathrm{ee}}-$ valor inicial (\$) dos equipamentos

O valor inicial da linha de alta tensão elétricos instalados.

$$
V i_{l a t}=L_{\text {lat. }} v u_{\text {lat }}
$$

Em que:

$\mathrm{L}_{\text {lat }}$ - comprimento da linha de alta tensão (m);

$\mathrm{vu}_{\text {lat }}$ - valor unitário médio $\left(\$ \mathrm{~m}^{-1}\right)$ da linha de alta tensão instalada.
$\mathrm{O}$ valor inicial dos equipamentos elétricos instalados $\left(\mathrm{Vi}_{\mathrm{ee}}\right)$ foi obtido a partir da equação 08 : 


$$
V i_{e e}=V i_{m e}+V i_{t e}+V i_{c p}+V i_{b c}+V i_{a e}+V_{\text {siee }}
$$

Em que:

$\mathrm{Vi}_{\mathrm{me}}$ - valor inicial (\$) do(s) motor(es) elétrico(s);

$\mathrm{Vi}_{\text {te }} \quad-\quad$ valor inicial (\$) $\operatorname{do}(\mathrm{s})$ transformador(es);

$\mathrm{Vi}_{\mathrm{cp}}$ - valor inicial (\$) do(s) comando(s) de partida;

$\mathrm{Vi}_{\mathrm{bc}}$ - valor inicial (\$) do banco de capacitores;

$\mathrm{Vi}_{\mathrm{ae}}$ - valor inicial (\$) dos acessórios elétricos;
$\mathrm{V}_{\text {siee }}$ - valor (\$) dos serviços de instalação dos equipamentos elétricos.

Para o motor à diesel, o valor inicial foi considerado como o somatório do custo de aquisição do motor, dos acessórios e a instalação.

Para a determinação do custo anual variável do sistema elevatório, foram utilizadas as equações $09,10,11$ e 12 para motor à diesel e as equações 09,10 e da 13 a 17 para motor elétrico:

$$
C A V=C A B+C A M
$$

Em que:

$\mathrm{CAB}$ - custo anual de bombeamento (\$); CAM - custo anual com manutenção e reparos $(\$)$.

$$
\text { Em que: } \quad C A M=\sum_{i=1}^{I} V i_{i} \cdot f m r_{i}
$$

$\mathrm{fmr}_{\mathrm{i}}$ - fração do valor inicial do i-ésimo componente gasto anualmente com sua manutenção e reparos.
O custo anual com manutenção e reparos (CAM) foi obtido conforme a equação 10 :
O custo anual de bombeamento $(\mathrm{CAB})$ para motor à diesel foi obtido conforme as equações 11 e 12:

$$
C B=\frac{Q \cdot H \cdot \gamma \cdot C U \cdot C_{u c} \cdot t}{735 \cdot \eta_{b h}}
$$

Em que:

CU - consumo unitário do motor à combustão $\left(\mathrm{m}^{3} \mathrm{cv}^{-1} \mathrm{~h}^{-1}\right)$; $\mathrm{C}_{\mathrm{uc}}$ - custo unitário do combustível $\left(\$ \mathrm{~m}^{-3}\right)$;

$\mathrm{t}$ - tempo de funcionamento do sistema elevatório (h); $\eta_{\mathrm{bh}}-$ rendimento da bomba

$$
C A B=\sum_{n=1}^{N} C B_{n}
$$

Em que:

$\mathrm{CB}_{\mathrm{n}}$ - custo de bombeamento do n-ésimo período de operação do sistema no ano.
O custo anual de bombeamento (CAB) para motor elétrico foi obtido conforme as equações 13 a 17 :

$$
C A B=F A D+F A C+A J A
$$

Em que:

FAD - faturamento anual da demanda (\$);
FAC - faturamento anual do consumo de energia elétrica (\$); 
AJA - ajuste anual referente ao fator de potência da instalação elétrica (\$).

O faturamento anual de demanda varia conforme a modalidade de aplicação de tarifas de energia elétrica. Neste estudo, foram levantadas as três modalidades existentes, convencional, tarifa verde e tarifa azul, sendo utilizada apenas a tarifa azul para confrontamento com o motor diesel, tendo em vista que esta modalidade é a mais econômica nas regiões analisadas (Monteiro et al., 2007). Portanto a equação 14 abaixo mostra como foi obtido o FAD:

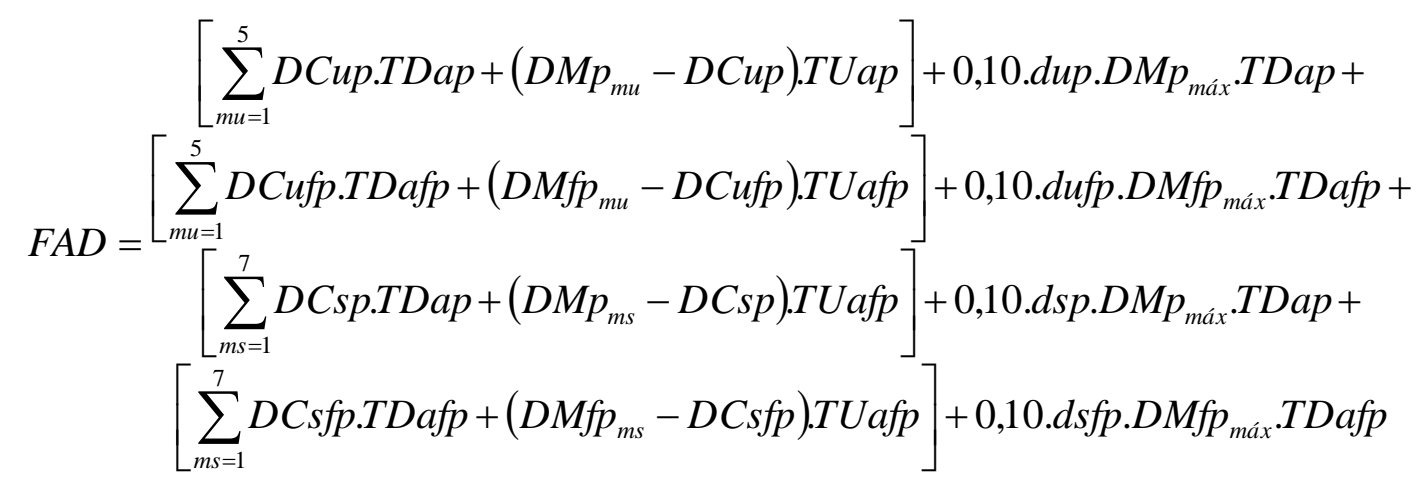

Em que:

DCup - demanda contratada no período úmido, no horário de ponta $(\mathrm{kW})$;

Dcufp - demanda contratada no período úmido, no horário fora de ponta $(\mathrm{kW})$, que não poderá ser inferior a DCup;

DCsp - demanda contratada no período seco, no horário de ponta $(\mathrm{kW})$, que não poderá ser inferior a DCup;

DCsfp - demanda contratada no período seco, no horário fora de ponta $(\mathrm{kW})$, que não poderá ser inferior a DCsp, e também não poderá ser superior a DCufp;

TDap - tarifa de demanda azul no horário de ponta $\left(\$ \mathrm{~kW}^{-1}\right)$;

TDafp - tarifa de demanda azul no horário fora de ponta $\left(\$ \mathrm{~kW}^{-1}\right)$;

TUap - tarifa de ultrapassagem de demanda azul no horário de ponta $\left(\$ \mathrm{~kW}^{-1}\right)$;

TUafp - tarifa de ultrapassagem de demanda azul no horário fora de ponta $\left(\$ \mathrm{~kW}^{-1}\right)$;

dup - número de meses completos que o sistema elevatório fica desligado no período úmido do ano no horário de ponta e, com isso, ocorre faturamento de demanda correspondente a $10 \%$ da maior demanda medida nos últimos 11 meses neste segmento (horário de ponta), ou seja
$\mathrm{DMp}_{\text {máx }}$ Porém se a Dcup for igual a zero, tal faturamento não ocorre; dufp - número de meses completos que o sistema elevatório fica desligado no período úmido do ano no horário fora de ponta e, com isso, ocorre faturamento de demanda correspondente a $10 \%$ da maior demanda medida nos últimos 11 meses neste segmento (horário fora de ponta), ou seja DMfp $_{\text {máx; }}$

dsp - número de meses completos que o sistema elevatório fica desligado no período seco do ano no horário de ponta e, com isso, ocorre faturamento de demanda correspondente a $10 \%$ da maior demanda medida nos últimos 11 meses neste segmento (horário de ponta), ou seja $\mathrm{DMp}_{\text {máx. }}$ Porém se a DCsp for igual a zero, tal faturamento não ocorre;

dsfp - número de meses completos que o sistema elevatório fica desligado no período seco do ano no horário fora de ponta e, com isso, ocorre faturamento de demanda correspondente a $10 \%$ da maior demanda medida nos últimos 11 meses neste segmento (horário fora de ponta), ou seja DMfp $_{\text {máx. }}$ Porém se a DCsfp for igual a zero, tal faturamento não ocorre; 
A demanda de potência é obtida pela Equação 15. É importante considerar os períodos úmido e seco, e os horários de ponta e fora de ponta.

Em que:

Qmáx $\mathrm{m}_{\mathrm{m}}$ - vazão máxima do sistema elevatório no m-ésimo mês do ano $\left(\mathrm{m}^{3} \mathrm{~s}^{-1}\right)$; $\mathrm{H}$ - altura manométrica do sistema elevatório no m-ésimo mês do ano (m); $\gamma-$ peso específico da água $\left(\mathrm{N} \mathrm{m}^{-3}\right)$;

$$
\begin{aligned}
& \sum_{m u=1}^{5} C M p_{m u} \cdot T C a u p+\left[C M f p c_{m u}+C_{\left.m h e_{m u} \cdot(1-f d t c)\right] \cdot T C a u f p}+\right. \\
& \sum_{m s=1}^{7} C M p_{m s} \cdot T C a s p+\left[C M f p c_{m s}+C M h e_{m s} \cdot(1-f d t c)\right] \cdot T C a s f p
\end{aligned}
$$

Em que:

$\mathrm{CMp}_{\mathrm{mu}}$ - consumo medido (kWh) no horário de ponta $(17$ às $21 \mathrm{~h}$ ou definido pela concessionária) do mu-ésimo mês do período úmido;

$\mathrm{CMfpc}_{\mathrm{mu}}$ - consumo medido $(\mathrm{kWh})$ no horário fora de ponta (horas complementares à de ponta) do mu-ésimo mês do período úmido;

$\mathrm{CMhe}_{\mathrm{mu}}$ - consumo medido (kWh) no horário especial de desconto para irrigantes (23 às 5 h.) no mu-ésimo mês do período úmido;

$\mathrm{CMp}_{\mathrm{ms}}$ - consumo medido ( $\mathrm{kWh}$ ) no horário de ponta $(17$ às $21 \mathrm{~h}$ ou definido pela concessionária) do mu-ésimo mês do período seco; $\eta_{\mathrm{b}}$ - rendimento da bomba hidráulica;

$\eta_{\mathrm{me}}-$ rendimento do motor elétrico.

O faturamento anual de consumo (FAC) na tarifa azul foi obtido pela equação 16:

$\mathrm{CMfpc}_{\mathrm{ms}}$ - consumo medido $(\mathrm{kWh})$ no horário fora de ponta (horas complementares à de ponta) do mu-ésimo mês do período seco;

$\mathrm{CMhe}_{\mathrm{ms}}$ - consumo medido (kWh) no horário especial de desconto no mu-ésimo mês do período seco;

TCaup - tarifa de consumo azul no período úmido, no horário de ponta $\left(\$ \mathrm{kWh}^{-1}\right)$;

TCaufp - tarifa de consumo azul no período úmido, no horário fora de ponta $\left(\$ \mathrm{kWh}^{-1}\right)$;

TCasp - tarifa de consumo azul no período seco no horário de ponta $\left(\$ \mathrm{kWh}^{-1}\right)$;

TCasfp - tarifa de consumo azul no período seco no horário fora de ponta $\left(\$ \mathrm{kWh}^{-1}\right)$.

Para o ajuste anual referente ao fator de potência da instalação elétrica na tarifa azul, foi utilizada a equação 17:

$$
A J A=\left[\begin{array}{c}
F A D-0,10(\text { dup.TDap }+ \text { dufp.TDafp }+ \text { dsp.TDap }+d s f p \cdot T D a f p)+ \\
\sum_{m u=1}^{5} C M p_{m u} \cdot T C a u p+\left[C M f p c_{m u}+C M h e_{m u} \cdot(1-f d t c)\right] \text { TCaufp }+ \\
\sum_{m s=1}^{7} C M p_{m s} \cdot T C a s p+\left[C M f p c_{m s}+C M h e_{m s} \cdot(1-f d t c)\right] \text { TCasfp }
\end{array}\right]\left(\frac{0,92}{\cos \varphi}-1\right)
$$

Em que:

fdtc - fração de desconto sobre tarifa de consumo $(0,7,0,8$ ou 0,9 , conforme a região do país);
Um software foi desenvolvido para facilitar a determinação do consumo energético nos 4 anos de cultivo do café irrigado, através das equações acima. 
Conhecido o custo total anual e o volume de água aplicado, determinou-se o custo de adução de cada $\mathrm{m}^{3}$ aplicado na cultura do café, em ambas as regiões (Triângulo Mineiro e Oeste da Bahia), nos três sistemas de irrigação (irrigação por pivô central, pivô central com emissor LEPA e por gotejamento) e nas duas fontes energéticas (elétrica e diesel).

\section{RESULTADOS E DISCUSSÃO}

A lâmina média de água recomendada, nos 4 anos de cultivo do café, para aplicação pelo sistema de irrigação por gotejamento $\left(\mathrm{SI}_{3}=1,2 \mathrm{cv} \mathrm{ha}{ }^{-1}\right)$ foi, em média, de $75 \%$ e $60 \%$ inferior a dos outros sistemas de irrigação (pivô central convencional $\mathrm{SI}_{1}=2,0 \mathrm{cv}$ ha $^{-1}$ e pivô central com LEPA $\mathrm{SI}_{2}=1,5 \mathrm{cv} \mathrm{ha}^{-1}$ ), para as regiões do Triângulo Mineiro e do Oeste da Bahia, respectivamente (Tabela 3). Isto demonstra que, independentemente das diferentes condições edafoclimáticas das regiões analisadas, o uso de sistemas de irrigação com relação de potência/área mais eficientes, proporciona significativa economia de água no cultivo do café, sendo um pouco menor a economia de água em regiões com demanda atmosférica elevada como no Oeste da Bahia. Os sistemas de irrigação com relação potência/área mais alta $\left(2,0 \mathrm{cv} \mathrm{ha}{ }^{-1}\right)$ apresentam, normalmente, valores de coeficiente de uniformidade de Christiansen (CUC) um pouco menores, o que certamente pode ter contribuido, neste estudo, para ampliar as diferenças de consumo de água com os sistemas com menor relação potência/área $\left(1,5\right.$ e 1,2 cv ha ${ }^{-}$ ${ }^{1}$ ), pois este parâmetro reflete a qualidade da uniformidade de água na área irrigada e, conseqüentemente, na lâmina a ser aplicada. Segundo Santana et al. (2004), em sistemas de irrigação por gotejamento o uso eficiente da água é potencializado pelo fato de, no primeiro ano de cultivo do cafeeiro, predominar a evaporação da água do solo sobre a transpiração da cultura.

A análise comparativa da necessidade hídrica da cultura, para as duas regiões, no sistema de irrigação por gotejamento, evidenciou valores consideravelmente maiores (76\% no $4^{\circ}$ ano) para a região Oeste da Bahia em relação ao $\mathrm{TM}$, com diferença de $484 \mathrm{~mm}$ para o $4^{\circ}$ ano. Portanto, o estudo minuncioso do consumo hídrico da cultura do café e o sistema de aplicação é extremamente importante nas diferentes áreas produtoras devido às consideráveis variações edafoclimáticas entre as regiões. Segundo Mantovani et al. (2004), como as regiões cafeeiras têm passado por veranicos prolongados, mesmo em regiões onde a soma da precipitação anual satisfaz as necessidades da cultura do café, é fundamental que se utilizem técnicas para evitar déficits hídricos elevados, comprometendo a produção. Conforme Rezende et al. (2010), a irrigação na fase de formação do cafeeiro, quando comparadas às condições de sequeiro, influenciaram significativamente nas variáveis diâmetro de copa, altura de planta e número de ramos plagiotrópicos totais.

O estudo do consumo hídrico nos diferentes estádios de desenvolvimento da cultura também merece atenção. Na Tabela 3 constata-se a grande variação da lâmina requerida entre os anos de cultivo do café. Conforme Arruda \& Grande (2003), com base em 16 anos de experimento com café, há evidências de que com o aumento da idade da cultura, existe aumento na sensibilidade ao estresse hídrico. Isto evidencia a importância do estudo de um adequado programa de manejo de irrigação para estas regiões, visando otimizar o uso de água.

$\mathrm{Na}$ região do Triângulo Mineiro, comparando-se os valores do volume total de água utilizado pelo sistema de irrigação por gotejamento $\left(\mathrm{SI}_{3}\right)$ em relação ao sistema 
de irrigação por pivô central com emissor LEPA $\left(\mathrm{SI}_{2}\right)$ e ao sistema pivô central convencional $\left(\mathrm{SI}_{1}\right)$, na fase adulta da cultura ( $4^{\circ}$ ano), observou-se uma economia de água de 55 e $348,4 \mathrm{mil} \mathrm{m}^{3}$ ano $^{-1}$, respectivamente, numa área irrigada de 100 ha. Isto demonstra que, na região do Triângulo Mineiro, o sistema $\mathrm{SI}_{3}$ proporcionou uma redução expressiva de $35,5 \%$ no consumo de água do café adulto ao substituir o sistema $\mathrm{SI}_{1}$. Ao substitui o $\mathrm{SI}_{2}$, houve redução de $8 \%$ no consumo hídrico. Já para a região do Oeste da Bahia observou-se uma economia de $28,7 \%$ e de $11,7 \%$, com a substituição do sistema $\mathrm{SI}_{3}$ pelos sistemas $\mathrm{SI}_{1}$ e $\mathrm{SI}_{2}$, respectivamente.

$\mathrm{O}$ bombeamento de água para irrigação da cultura do café, usando motor diesel, representou um acréscimo médio de $170,9 \%$ e de $270,1 \%$ em relação ao acionamento elétrico nas regiões do Triângulo Mineiro e Oeste da Bahia, respectivamente. Como não foi considerada diferença nos custos unitários do diesel para as regiões, estes valores expressam que o acionamento elétrico na região do Oeste da Bahia é menos oneroso do que a região do Triângulo Mineiro (Tabela 3).

$\mathrm{O}$ uso do sistema $\mathrm{SI}_{3}$ na região do Triângulo Mineiro apresentou uma economia média de energia elétrica de $29 \%$ e $47,6 \%$ em relação aos sistemas 2 e 1 , respectivamente. Numa lavoura de 100 ha de café irrigado, utilizando a tarifa horosazonal azul, para região do Triângulo Mineiro essa economia no ano agrícola corresponderia a R\$ 8.249,98 e R \$ 29.429,06, respectivamente. Para a região do Oeste da Bahia utilizando a tarifa horosazonal azul, esta economia seria de $\mathrm{R} \$ 3.149,64$ e $\mathrm{R} \$$ 19.917,48. Quanto às composições tarifárias para as regiões do Triângulo Mineiro e Oeste da Bahia, a tarifa azul mostrou-se mais econômica aos irrigantes. Em ambas as regiões, a tarifa convencional não é recomendada face ao seu elevado custo.
Utilizando a tarifa horosazonal azul, observou-se que o custo (em reais) do metro cúbico de água aduzido decresceu com a idade da cultura, nas duas regiões estudadas, tanto com acionamento elétrico como a diesel (Figura 1a, 1b, 1c e 1d). Para a região do Triângulo Mineiro, em ambas as formas de bombeamento, o sistema 2 apresentou maior custo unitário de adução de água (Figura 1a, 1b). Isto demonstra que, mesmo com a economia de água de 293,4 mil m ano $^{-1}$ ao se optar pelo sistema 2 em relação ao sistema 1, ainda assim há um acréscimo de $\mathrm{R} \$ 2.462,70$ no custo anual de bombeamento elétrico. Já ao se optar pelo sistema 3 em detrimento do sistema 1 , além da economia de água de $348,4 \mathrm{mil} \mathrm{m}^{3} \mathrm{ano}^{-1}$, há uma economia de $\mathrm{R} \$ 20.241,00$ no ano agrícola. Para a região do Oeste da Bahia o sistema 3 apresentou maior custo do $\mathrm{m}^{3}$ aduzido (Figura 1c, 1d), no entanto, como esse sistema proporcionou uma economia de água de 450,7 mil m ano $^{-1}$ em relação ao sistema 1, foi o que apresentou menor custo anual de bombeamento elétrico (R\$ 59.201,00) comparado aos demais sistemas, apesar de a diferença para o sistema 2 ser praticamente insignificante no ano $(\mathrm{R} \$$ 249,30), sugerindo o uso do sistema 3, por ser mais interessante do ponto de vista ambiental.

Fazendo um comparativo entre as regiões (Figura 2.1, 2.2 e 2.3), observou-se que o custo médio unitário de adução de água para a região do Triângulo Mineiro foi superior a região do Oeste da Bahia nos três sistemas de irrigação. Este aumento, ao se optar não irrigar a região do Oeste da Bahia para irrigar a região do Triângulo Mineiro foi, em média, de $32,4 \%, 88,9 \%$ e $27,2 \%$, para o sistemas 1, 2 e 3, respectivamente. Observa-se que, no sistema 2 , o acréscimo foi bem expressivo. 
Tabela 3. Consumo de água e custo total horário ${ }^{1}$ estimado do bombeamento de água para irrigação do cafeeiro, em quatro anos de cultivo, irrigado por três sistemas de irrigação numa área de 100 ha, nas regiões do Triângulo Mineiro e do Oeste da Bahia.

\begin{tabular}{|c|c|c|c|c|c|c|c|c|}
\hline \multicolumn{5}{|c|}{ TRIÂNGULO MINEIRO } & \multicolumn{4}{|c|}{ OESTE DA BAHIA } \\
\hline Ano & $\begin{array}{c}\text { Tempo } \\
\text { total de } \\
\text { irrigação } \\
\text { (h) }\end{array}$ & $\begin{array}{c}\text { Lâmina } \\
\text { total } \\
(\mathbf{m m})\end{array}$ & $\begin{array}{c}\text { Tarifa } \\
\text { Azul }^{2} \\
(\mathbf{R} \$)\end{array}$ & $\begin{array}{c}\text { Diesel } \\
(\mathbf{R} \$)\end{array}$ & $\begin{array}{c}\text { Tempo } \\
\text { total de } \\
\text { irrigação } \\
\text { (h) }\end{array}$ & $\begin{array}{c}\begin{array}{c}\text { Lâmina } \\
\text { total }\end{array} \\
(\mathbf{m m})\end{array}$ & $\begin{array}{c}\text { Tarifa } \\
\text { Azul }^{2} \\
(\mathbf{R} \$)\end{array}$ & $\begin{array}{c}\text { Diesel } \\
(\mathbf{R} \$)\end{array}$ \\
\hline \multicolumn{9}{|c|}{ Pivô Central Convencional $\left(S I_{1}=2,0 \mathrm{cv} \mathrm{ha^{-1 } )}\right.$} \\
\hline 1 & 932,7 & 427,8 & 54,66 & 130,56 & $1.485,0$ & 783,4 & 32,77 & 118,83 \\
\hline 2 & $1.535,5$ & 705,1 & 38,47 & 118,17 & $2.284,0$ & $1.202,0$ & 24,61 & 111,89 \\
\hline 3 & $2.043,6$ & 910,6 & 32,35 & 113,41 & $2.945,1$ & $1.522,1$ & 21,80 & 109,00 \\
\hline 4 & $2.206,8$ & 981,5 & 30,91 & 112,34 & $3.012,5$ & $1.567,7$ & 21,75 & 108,77 \\
\hline \multicolumn{9}{|c|}{ Pivô Central LEPA $\left(S I_{2}=1,5 c v h a^{-1}\right)$} \\
\hline 1 & $1.031,6$ & 200,5 & 40,15 & 95,65 & $1.162,0$ & 413,7 & 30,72 & 93,25 \\
\hline 2 & $1.904,3$ & 370,2 & 26,23 & 85,85 & $1.870,2$ & 648,2 & 21,96 & 86,06 \\
\hline 3 & $2.786,6$ & 541,2 & 21,08 & 82,17 & $2.985,2$ & $1.020,7$ & 17,02 & 81,65 \\
\hline 4 & $3.539,9$ & 688,1 & 19,89 & 80,49 & $3.623,6$ & $1.264,9$ & 16,55 & 80,34 \\
\hline \multicolumn{9}{|c|}{ Gotejamento $\left(\mathrm{SI}_{3}=1,2 \mathrm{cv} \mathrm{ha^{-1 }}\right)$} \\
\hline 1 & 805,2 & 187,2 & 40,78 & 81,34 & $1.294,2$ & 347,5 & 24,02 & 73,05 \\
\hline 2 & $1.308,0$ & 313,4 & 27,96 & 72,90 & $2.087,0$ & 551,9 & 17,24 & 67,86 \\
\hline 3 & $2.086,7$ & 503,4 & 20,65 & 67,87 & $3.704,4$ & 922,7 & 13,65 & 64,17 \\
\hline 4 & $2.693,5$ & 633,1 & 17,63 & 65,96 & $4.488,7$ & $1.117,0$ & 13,27 & 63,34 \\
\hline
\end{tabular}

${ }^{\mathrm{T}}$ Custo horário de aplicação (variável) + custo horário fixo (motor + rede elétrica) ${ }^{2}{ }^{2}$ Tarifa mais econômica comparada às tarifas verde e convencional obtidas nas concessionárias energéticas: Companhia Energética de Minas Gerais (CEMIG) (Resolução 083/2004) e Companhia de Eletricidade do Estado da Bahia (COELBA) (Resolução 319/2006) 


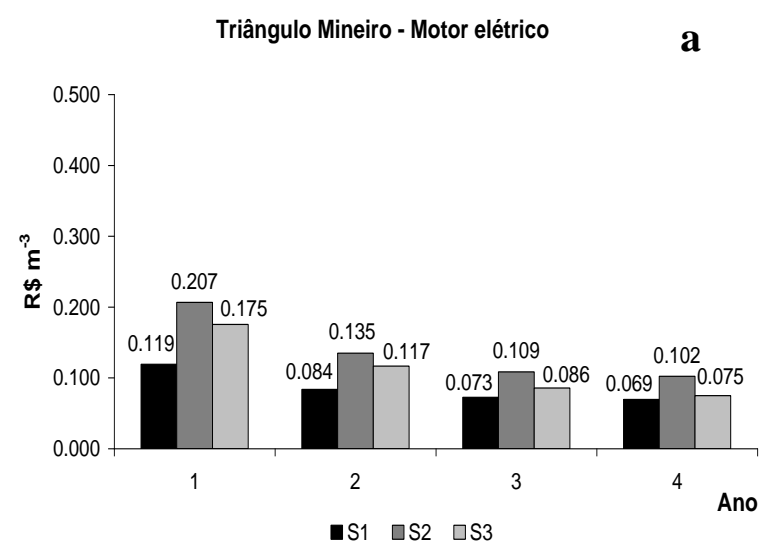

Oeste da Bahia - Motor elétrico

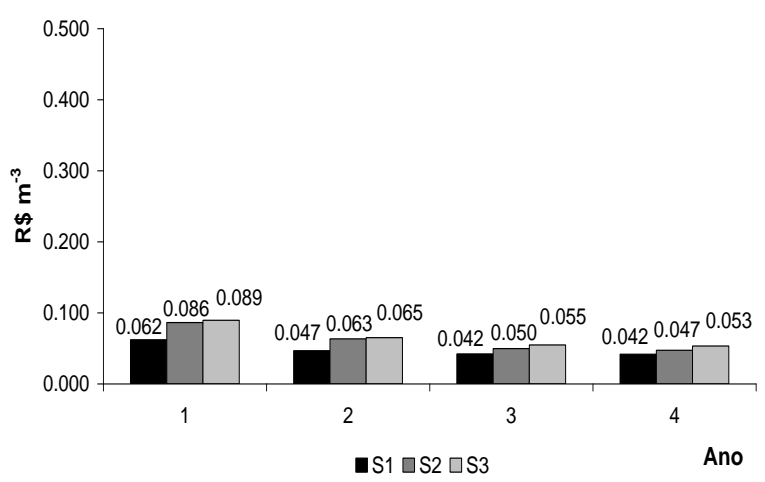

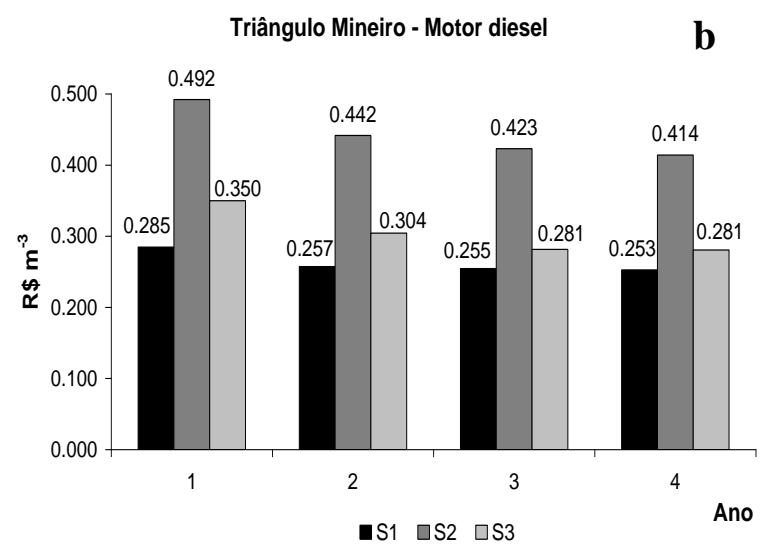

Oeste da Bahia - Motor diesel

d

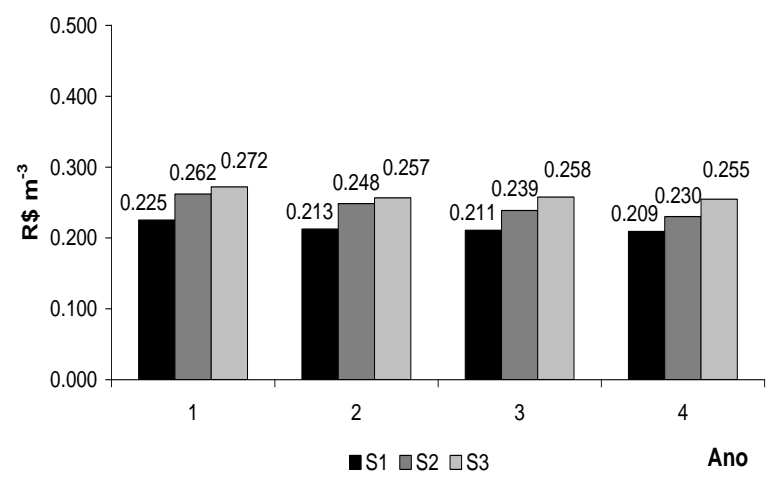

Figura 1. Custo, em reais, do $\mathrm{m}^{3}$ de água aduzida para o café, nos quatro primeiros anos da cultura, utilizando motor elétrico e diesel, nos sistemas de irrigação por pivô central convencional $\left(\mathrm{SI}_{1}\right)$, por pivô central com emissor LEPA $\left(\mathrm{SI}_{2}\right)$ e irrigação por gotejamento $\left(\mathrm{SI}_{3}\right)$, nas regiões do Triângulo Mineiro (a, b) e do Oeste da Bahia (c, d).


Figura 2. Valor médio do custo, em reais, do $\mathrm{m}^{3}$ de água aduzida para o café, em três sistemas de irrigação (1, 2 e 3), nos quatro primeiros anos da cultura, nas regiões do Triângulo Mineiro (TM) e do Oeste da Bahia (OB). 


\section{CONCLUSÕES}

Em ambas as regiões (Triângulo Mineiro e Oeste da Bahia), numa área de café irrigado, o sistema de irrigação por gotejamento utiliza menos água e energia em um ano de produção. Já o custo, em reais, da adução de um $\mathrm{m}^{3}$ de água pelo sistema de gotejamento, no Oeste da Bahia, é maior em ambas fontes de energia (elétrica ou diesel). Para a região do Triângulo Mineiro, o sistema de irrigação por pivô central com emissor LEPA apresenta maior custo unitário de adução de água.

\section{REFERÊNCIAS BIBLIOGRÁFICAS}

ALLEN, R.G.; PEREIRA, L. S.; RAES, D.; SMITH, M. 1998. Guidelines for computing crop water requeriments. Rome: FAO. 308p. (FAO Irrigation and Drainage, 56).

ALVES JÚNIOR, J.; FIGUEREDO, L.; COELHO, R.D.; ZOCOLER, J.L. 2003. Quanto custa a energia elétrica na irrigação. Anuário da Agricultura Brasileira: AGRIANUAL 2004, São Paulo, p.19-22.

ARRUDA, F.B., GRANDE, M.A. 2003. Fator de resposta da produção do cafeeiroao deficit hídrico em Campinas. Bragantia, Campinas, v.62, n.1, p.139145.

BRASIL. Agência Nacional de Energia Elétrica. 2002. Resolução n. 207 de 19 de julho de 2000. Diário Oficial da União, Brasília, DF, 18 abr., Seção 1, p.107, v.139, n. 74.

BRASIL. Departamento Nacional de Águas e Energia Elétrica. 2002. Portaria n. 105 de 03 de abril de 2002. Diário Oficial da União, Brasília, DF, 06 abr., Seção 1, p.4321.

BONOMO, R. Análise da irrigação na cafeicultura em áreas de cerrado de
Minas Gerais. Viçosa - MG: UFV, 1999. 224 p. Tese (Doutorado em Engenharia Agrícola) - Universidade Federal de Viçosa, 1999.

CEMIG - Companhia Energética de Minas Gerais. Resolução 83/2004. 2006. Disponível em: <http://www.cemig.com.br>. Acesso em: 12 outubro.

COELBA - Companhia de Eletricidade do Estado da Bahia. 2006. Resolução 319/2006. Disponível em: <http://www.coelba.com.br>. Acesso em: 12 outubro.

EMBRAPA - Empresa Brasileira de Pesquisa Agropecuária. Centro Nacional de Pesquisa de Solos. Manual de métodos de análise de solos. 2. ed. Rio de Janeiro, 1997. 212 p.

GOMES, N. M.; LIMA, L.A.; CUSTODIO, A.A.P. 2007. Crescimento vegetativo e produtividade do cafeeiro irrigado no sul do Estado de Minas Gerais. Revista Brasileira de Engenharia Agrícola e Ambiental, Campina Grande, v.11, n.6, p.564-570.

IRRIGER. Gerenciamento e Engenharia de Irrigação. Viçosa, 2006. Disponível em: < http://www.irriger.com.br/index.php> . Acesso em: 17 de dezembro 2009.

KELLER, J., KARMELI, D. 1975. Trickle irrigation design. Glendora: Rain Bird Sprinkler Manufacturing. 133p.

LIMA, L.A; CUSTODIO, A.A.P.; GOMES, N.M. 2008. Produtividade e rendimento do cafeeiro nas cinco primeiras safras irrigado por pivô central em Lavras, MG. Ciências Agrotécnicas, Lavras, v. 32, n. 6, p. 1832-1842, nov./dez.

MANTOVANI, E.C.; VICENTE, M.R.; SOUZA, M.N. Caracterização técnica e perspectivas para a cafeicultura irrigada brasileira. In: ZAMBOLIM, L. (Org.). Efeitos da irrigação sobre a qualidade e produtividade do café. Viçosa - MG: UFV, 2004. v.1, p.293-318. 
MONTEIRO, R.O.C. FERRAZ, P.; COELHO, R.D.; SANTOS, R.A. 2007. Distância da rede elétrica que viabiliza o uso de motores diesel em áreas irrigadas do Brasil. Irriga, Botucatu, v. 12, n. 2, p. 263-272.

REZENDE, F.C. OLIVEIRA, S.R. ; FARIA, M.A.; ARANTES, RESENDE, K. 2006. Características produtivas do cafeeiro (Coffea arabica L. cv., Topázio MG1190), recepado e irrigado por gotejamento. Coffee Science, Lavras, v.1, n.2, p.103-110, jul./dez.

REZENDE, R.; HELBEL JÚNIOR, C.; SOUZA, R. S.; ANTUNES, F. M.; FRIZZONE, J. A. 2010. Crescimento inicial de duas cultivares de cafeeiro em diferentes regimes hídricos e dosagens de fertirrigação. Engenharia Agrícola, Jaboticabal, v. 30, n. 3, p. 447-458.

SANTANA, M.S.; OLIVEIRA, C.A.S.; QUADROS, M. Crescimento inicial de duas cultivares de cafeeiro adensado influenciado por níveis de irrigação localizada. Engenharia Agrícola, Jaboticabal, v.24, n.3, p.644-653, 2004.
SANTINATO, R.; FERNANDES, A.L.T. Cultivo do cafeeiro irrigado em plantio circular sob pivô central, BeloHorizonte, Editora O Lutador, 2002, 251 p.

SILVA, A.M.; LIMA, E.P.; COELHO, G.S.; COELHO, M.R. 2003. Produtividade, rendimento de grãos e comportamento hídrico foliar em função da época, parcelamento e do método de adubação do cafeeiro Catuaí. Engenharia Agrícola, Jaboticabal, v.23, n.3, p.434440,set./dez.

SILVA, A.M.; SILVA, P.A. M.; FARIA, M. A.; GUIMARÂES, P.T.G.; COELHO, M.R.; COELHO, G.S. 2002. Avaliação do efeito da época de irrigação e da fertirrigação sobre a produtividade e qualidade do café (safra 1999/2000). Engenharia Agrícola, Jaboticabal, v. 22, n. 3, p. 312-321, set.

SOUZA, L.O.C. Análise técnica de sistemas de irrigação por gotejamento utilizados na cafeicultura irrigada. Viçosa. 98p. Dissertação (Mestrado em Engenharia Agrícola). Departamento de Engenharia Agrícola - UFV, 2000. 